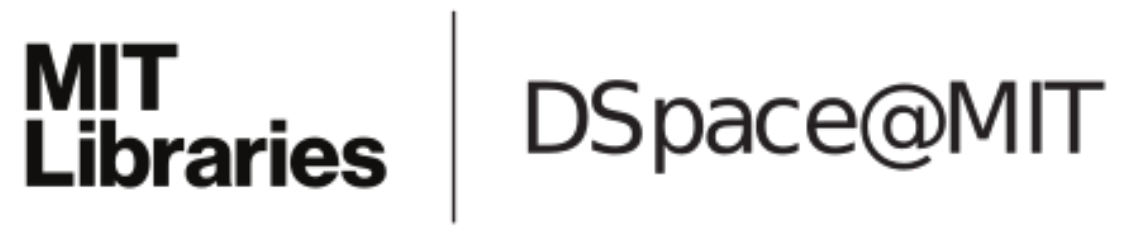

\author{
MIT Open Access Articles
}

Maximum flows and minimum cuts in the plane

The MIT Faculty has made this article openly available. Please share how this access benefits you. Your story matters.

Citation: Strang, Gilbert. "Maximum Flows and Minimum Cuts in the Plane." Journal of Global Optimization 47, 3 (September 2009): 527-535 ๔ 2009 Springer Science+Business Media

As Published: http://dx.doi.org/10.1007/s10898-009-9471-6

Publisher: Springer-Verlag

Persistent URL: http://hdl.handle.net/1721.1/116027

Version: Author's final manuscript: final author's manuscript post peer review, without publisher's formatting or copy editing

Terms of use: Creative Commons Attribution-Noncommercial-Share Alike 


\title{
Maximum Flows and Minimum Cuts in the Plane
}

\author{
Gilbert Strang
}

\begin{abstract}
A continuous maximum flow problem finds the largest $t$ such that $\operatorname{div} v=t F(x, y)$ is possible with a capacity constraint $\left\|\left(v_{1}, v_{2}\right)\right\| \leq c(x, y)$. The dual problem finds a minimum cut $\partial S$ which is filled to capacity by the flow through it. This model problem has found increasing application in medical imaging, and the theory continues to develop (along with new algorithms). Remaining difficulties include explicit streamlines for the maximum flow, and constraints that are analogous to a directed graph.
\end{abstract}

Keywords Maximum flow, Minimum cut, Capacity constraint, Cheeger

\section{Introduction}

This paper returns to a special class of problems (partial differential equations with inequality constraints) in continuous linear programming. They describe flow through a domain $\Omega$, in analogy with flow along the edges of a graph. The flow is maximized subject to a capacity constraint. The key to the solution is the dual problem, which looks for a set $S \subset \Omega$ from which no more flow is possible. The boundary of $S$ is the minimum cut, and it is filled to capacity by the maximum flow.

In the discrete case, Kirchhoff's Current Law that "flow in = flow out" must hold at every interior node of the network. The maximum flow is the largest flow from source to sink, subject to Kirchhoff's equation at the nodes and capacity constraints on the edges. This fits the standard framework of linear programming, and Kirchhoff's incidence matrix (of 1's, -1's, and 0's) has remarkable properties that lead to an attractive theory. Our purpose is to point to a maximum flow-minimum cut theorem in the continuous case, and to introduce new questions.

The principal unknown is the vector $v(x, y)$ that gives the magnitude and direction of the flow. On a plane domain this is $v=\left(v_{1}(x, y), v_{2}(x, y)\right)$. The analog of Kirchhoff's matrix is the divergence operator:

$$
\text { Conservation } \quad \operatorname{div} v=\frac{\partial v_{1}}{\partial x}+\frac{\partial v_{2}}{\partial y}=t F(x, y) \quad \text { in } \Omega .
$$

That source/sink term $t F(x, y)$ might be zero or nonzero in the interior of the flow domain $\Omega$. There may also be a source term $t f(x, y)$ on the boundary $\partial \Omega$, 
in closer analogy with the discrete source and sink in classical network flow. With $n$ as the unit normal vector to $\partial \Omega$, the (possible) boundary sources and sinks are given by a Neumann condition:

$$
\text { Boundary sources } v \cdot n=t f(x, y) \text { on } \partial \Omega_{N} \text {. }
$$

Our examples will involve $F$ but not $f$. We only note the case $\partial \Omega_{N}=\partial \Omega$, when $f$ is prescribed on the whole boundary. Then the divergence theorem $\iint \operatorname{div} v d x d y=\int v \cdot n d s$ imposes a compatibility condition on $F$ and $f$ :

$$
\text { Compatibility } \iint_{\Omega} F(x, y) d x d y=\int_{\partial \Omega} f(x, y) d s \quad \text { if } \quad \partial \Omega_{N}=\partial \Omega .
$$

Now comes the key inequality, a limit on the flow. The vector field $v(x, y)$ is subject to a capacity constraint, which makes the problem nonlinear. In our original paper [31] this constraint measured $\left(v_{1}, v_{2}\right)$ always in the $\ell^{2}$ norm at each point:

$$
\text { Capacity }|v(x, y)|=\sqrt{v_{1}^{2}+v_{2}^{2}} \leq c(x, y) \text { in } \Omega .
$$

A more general condition would require $v(x, y)$ to lie in a convex set $K(x, y)$ :

$$
v(x, y) \in K(x, y) \text { for all } x, y \text { in } \Omega .
$$

A typical maximal flow problem in the domain $\Omega$ is

Maximize $t$ subject to (1), (2), and (4).

In returning to this maximal flow problem, our goal is to highlight four questions that were not originally considered. Fortunately there has been good progress by several authors, and partial answers are available. But the new tools are not yet all-powerful, as we illustrate with a challenge problem (uniform source $F=1$ and capacity $c=1$ with $\Omega=$ unit square). This continues to resist explicit solution for the velocity vector $v$ :

Challenge Maximize $t$ so that $\operatorname{div} v=t$ with $|v| \leq 1$ in $\Omega$.

The intriguing aspect of this problem is that we can identify the minimal cut. Therefore we know the maximal flow factor $t=2+\sqrt{\pi}$, from the capacity across that cut $\partial S$. Determining $\partial S$ is a constrained isoperimetric problem that is pleasant to solve (and raises new questions).

What we do not know is the flow vector $v$ inside the square! Optimality tells us the magnitude and direction of $v$ only along the cut, described below. We apologize for the multiplication of new challenges, when the proper goal of a paper should be new solutions.

\section{New Questions and Applications}

The continuous maximal flow problem is attracting a small surge of interest. We mention recent papers that carry the problem forward in several directions: 
1. Grieser [16] shows how max flow-min cut duality leads to an elegant proof of Cheeger's inequality, giving the lower bound in (18) on the first eigenvalue of the Laplacian on $\Omega$. The eigenfunction has $u=0$ on $\partial \Omega$, so $\partial \Omega_{N}$ is empty:

$$
\text { Cheeger } \quad \lambda_{1} \geq \frac{1}{4} h^{2} \quad \text { where } h(\Omega)=t_{\max } \text { with } F \equiv 1 \text {. }
$$

The Cheeger constant $h$ is found from the constrained isoperimetric problem that arises for the minimal cut $\partial S$ :

$$
\text { Definition } h(\Omega)=\inf _{S \subset \Omega} \frac{\text { perimeter of } S}{\text { area of } S} .
$$

As in the particular case of our challenge problem, $h(\Omega)$ is often computable. For the unit square we will note in (24) that the inequality (7) is far from tight.

2. Appleton and Talbot [2] have proposed an algorithm for computing the maximum flow vector $v$ from a sequence of discrete problems. Their motivation is to study image segmentation with medical applications (see especially [3, 4]). The same techniques are successful in stereo matching [26]. Their paper is rich in ideas for efficient computations and an excellent guide to the literature.

The algorithm approaches the maximum flow field as $T \rightarrow \infty$, by introducing a "Maxwell wave equation" with capacity $c$ and internal source $F=0$ :

$$
\text { Appleton-Talbot } \quad \frac{\partial E}{\partial T}=-\operatorname{div} v, \quad \frac{\partial v}{\partial T}=-\operatorname{grad} E, \quad|v| \leq c .
$$

The potential is $E$, and the first equation $\partial E / \partial T=-\operatorname{div} v$ is a relaxation of the conservation constraint $\operatorname{div} v=0$ (Kirchhoff's Law). Appleton and Talbot prove that the energy $\iint\left(|E|^{2}+|v|^{2}\right)$ is decreasing in every subset $S$ of $\Omega$. At convergence, the optimal cut is the boundary of a level set of $E$.

The equations (9) are discretized on a staggered grid. This corresponds to Yee's method (also called the FTDT method) in electromagnetics. The algorithm has a weighting function to model the effect of source terms, and the experiments with image segmentation are very promising.

Since primal-dual interior point algorithms have become dominant in optimization, we conjecture that those methods can be effective also here in the approximation of continuous by discrete maximal flows.

3. Nozawa [23] took a major step in extending the max flow-min cut theorem from the simple isotropic condition $|v| \leq 1$ in (4) toward the much more general capacity condition (5). This step can be illustrated already in our challenge problem, by changing from the $\ell^{2}$ norm of $v(x, y)$ to the $\ell^{1}$ or $\ell^{\infty}$ norm:

$\ell^{\mathbf{1}}$ challenge Maximize $t$ so that $\operatorname{div} v=t$ with $\left|v_{1}\right|+\left|v_{2}\right| \leq 1$ in $\Omega$

$\ell^{\infty}$ challenge Maximize $t$ so that $\operatorname{div} v=t$ with $\left|v_{1}\right| \leq 1,\left|v_{2}\right| \leq 1$ in $\Omega$ 
In the isoperimetric problem (8), this changes the definition of perimeter. The dual norm (in this case $\ell^{\infty}$ or $\ell^{1}$ ) becomes the measure of arclength $(d x, d y)$. Then this dual norm enters the computation of $|\partial S|$ :

$$
\text { Perimeter (in } \mathbf{R}^{2} \text { ) } \quad|\partial S|=\int_{\partial S}|(d x, d y)| .
$$

The coarea formula from geometric measure theory [12], on which the proof of duality rests, continues to apply with the new definition.

As in the $\ell^{2}$ case, the maximal $t$ can be computed! So we have new flow fields to find, reaching bounds that duality says are achievable.

It is intriguing to connect maximal flow with the other central problem for networks and continua - the transportation problem. This asks for shortest paths. The original work of Monge and Kantorovich on continuous flows has been enormously extended by Evans [11], Gangbo and McCann [15], Rachev and Rüschendorf [25], and Villani [33].

Our challenge problem requires the movement of material $F(x, y)$ from $\Omega$ to $\partial \Omega$. The bottleneck is in moving from the interior of $S$ to the minimal cut $\partial S$. The distribution of material is uniform in $S$, and its destination is uniform along $\partial S$, to use all the capacity allowed by $|v| \leq 1$. How is the shortest path (Monge) flow from $S$ to $\partial S$ related to the maximum flow?

4. Directed graphs and flows. Chung $[8,9]$ has emphasized that Cheeger's theory (and the Laplacian itself) are not yet fully developed for directed graphs. For maximal flow on networks, Ford and Fulkerson [13] had no special difficulty when the edge capacities depend on the direction of flow. The problem is still a linear program and duality still holds.

For directed continuous flows we lack a correctly formulated duality theorem. The capacity would be a constraint $v(x, y) \in K(x, y)$ as in (5). Nozawa's duality theorem in [23] quite reasonably assumed that zero is an interior point of $K$. Then a flow field exists for sufficiently small $t$ (the feasible set is not empty). The continuous analog of direction-dependent capacities seems to require analysis of more general convex sets $K(x, y)$, when zero is a boundary point. In [22], Nozawa illustrated duality gaps when his hypotheses were violated.

Finally we mention that all these questions extend to domains $\Omega$ in $\mathbf{R}^{n}$. The constrained isoperimetric problems generalize to higher dimensions as well as different norms. The one simplification in the plane is the introduction of a stream function $s(x, y)$, with $\left(v_{1}, v_{2}\right)=(\partial s / \partial y,-\partial s / \partial x)$ as the general solution to $\operatorname{div} v=0$. Our survey [30] formulated the corresponding primal and dual problems for $s(x, y)$ as $L^{1}$ and $L^{\infty}$ approximations of planar vector fieldswhere Laplace's equation corresponds to $L^{2}$.

The remaining sections of this paper discuss the topics outlined above. We compute the minimum cuts in the three versions of the challenge problem on the unit square. We also mention an isoperimetric problem (with a different definition of perimeter) to which we return in a later paper [32]. 


\section{Duality, Coarea, and Cheeger Constants}

The maximum flow is limited by the capacity $c(x, y)$ :

Primal problem Maximize $t$ subject to

$\operatorname{div} v=t F(x, y)$ in $\Omega, \quad v \cdot n=t f(x, y)$ on $\partial \Omega_{N}, \quad|v(x, y)|_{2} \leq c(x, y)$ in $\Omega$.

Nozawa's duality theorem requires a proper choice of function spaces and boundary conditions, in this problem and in its dual for $u(x, y)$ in $\mathrm{BV}(\Omega)$. Where the primal involves the divergence, the dual involves the gradient. Kohn and Temam [19] extended Green's formula $\iint u \operatorname{div} v=-\iint v \cdot \operatorname{grad} u$ to allow functions $u(x, y)$ of bounded variation.

We will see that the optimal $u(x, y)$ in the dual problem is the characteristic function of a set $S$ with finite perimeter. This $u(x, y)$ is not smooth, but it lies in BV. The dual problem does not initially ask for a minimum cut!

Dual problem Minimize $\|u\|_{\mathrm{BV}, c}$ with $\ell(u)=1 \quad$ OR $\quad$ Minimize $\frac{\|u\|_{\mathrm{BV}, c}}{|\ell(u)|}$

$$
\|u\|_{\mathrm{BV}, c}=\iint_{\Omega} c(x, y)|\operatorname{grad} u|_{2} d x d y \quad \ell(u)=\int_{\partial \Omega_{N}} u f d s-\iint_{\Omega} u F d x d y .
$$

The key step toward the solution is to recognize the extreme points of the unit ball in this weighted BV norm $\|u\|_{\mathrm{BV}, c}=\iint c|\operatorname{grad} u|_{2} d x d y$. Those extreme points are characteristic functions $u=\chi_{S}$ of open subsets $S$ of $\Omega$ :

$$
\chi_{S}(x, y)=\{1 \text { for } x, y \text { in } S, \quad 0 \text { otherwise }\} .
$$

The BV norm of $\chi_{S}$ is the weighted perimeter $\int c d s$ of $S$, because the gradient is a measure (a line of delta functions) supported only on that boundary $\partial S$.

The coarea formula gives the BV norm of $u$ (weighted by the capacity $c$ ) as an integral over the norms of characteristic functions of level sets $S(t)$ of $u$ :

$$
\text { Coarea }\|u\|_{\mathrm{BV}, c}=\int\left\|\chi_{S(t)}\right\|_{\mathrm{BV}, c} d t \quad \text { with } \quad S(t)=\{x, y \mid u(x, y)<t\} .
$$

Consider the case with $F \geq 0$ and no boundary sources $f$. Specializing in (13) to the characteristic functions $u=\chi_{S}$, our dual problem reduces to an isoperimetric problem for $S$ and the minimum cut $\partial S$ :

$$
\min _{u \in \mathrm{BV}} \frac{\iint c|\operatorname{grad} u|_{2} d x d y}{\iint F u d x d y}=\min _{S \subset \Omega} \frac{\text { weighted perimeter } \int_{\partial S} c d s}{\text { weighted area } \iint_{S} F d x d y} .
$$

Choosing $c(x, y)=1$ and $F(x, y)=1$, this computes the Cheeger constant:

$$
\text { Cheeger constant } h(\Omega)=\inf _{S \subset \Omega} \frac{|\partial S|}{|S|} .
$$


Weak duality $h \geq t$ is the inequality $\int c d s \geq t \iint F d x d y$ for every feasible $t$ in the primal problem. This is just the divergence theorem when $\operatorname{div} v=t F(x, y)$ and $|v| \leq 1$ :

$$
\underset{\boldsymbol{h} \geq \boldsymbol{t}}{\text { Weak duality }} \quad \int_{\partial S} c d s \geq \int_{\partial S} v \cdot n d s=\iint_{S} \operatorname{div} v d x d y=t \iint_{S} F d x d y .
$$

Duality says that equality holds for the maximal flow $v$ and the minimal cut $\partial S$.

Historically, the key inequality given by Cheeger [7] was a lower bound on the first eigenvalue $\lambda_{1}$ of the Laplacian on the domain $\Omega$. Grieser [16] observed how neatly and directly this bound follows from Green's formula, when $F=1$ and $|v| \leq 1$. We expect to see the Schwarz inequality in the step from problems in $L^{1}$ and $L^{\infty}$ to the eigenvalue problem in $L^{2}$ :

$$
\begin{aligned}
t \iint u^{2} & =\int(\operatorname{div} v) u^{2}=-\iint v\left(\operatorname{grad} u^{2}\right) \\
& \leq 2 \iint|u||\operatorname{grad} u| \leq 2\left[\iint u^{2} \iint|\operatorname{grad} u|^{2}\right]^{1 / 2} .
\end{aligned}
$$

Thus any feasible $t$ gives a lower bound $t^{2} / 4$ to the Rayleigh quotient for any $u(x, y)$ with $u=0$ on $\partial \Omega$ :

$$
\frac{t^{2}}{4} \leq \frac{\iint|\operatorname{grad} u|^{2} d x d y}{\iint u^{2} d x d y}
$$

The minimum of the right side is $\lambda_{1}(\Omega)$, and the maximum of the left side is $h^{2} / 4$. Cheeger's inequality becomes $h^{2} / 4 \leq \lambda_{1}(\Omega)$.

A widely studied paper [10] of Diaconis and Stroock introduces another very useful measure of the "bottleneck" that limits flow on a graph.

\section{The Challenge Problems}

When we use the $\ell^{2}$ norm of the flow vector $v=\left(v_{1}, v_{2}\right)$ at each point, the constraint $|v(x, y)| \leq c(x, y)$ is isotropic. Other norms of $v$ give constraints that come closer to those on a discrete graph. The edges of the graph might be horizontal and vertical (from a square grid) or at $45^{\circ}$ and $-45^{\circ}$ (from a staggered grid). We will use the challenge problem with $F=c=1$ on a unit square as an example that allows computation of the minimal cut in all three cases:

$$
\begin{array}{lr}
\ell^{2} \text { constraint } & v_{1}^{2}+v_{2}^{2} \leq 1 \\
\ell^{1} \text { constraint } & \left|v_{1}\right|+\left|v_{2}\right| \leq 1 \\
\ell^{\infty} \text { constraint } & \max \left(\left|v_{1}\right|,\left|v_{2}\right|\right) \leq 1
\end{array}
$$

The $\ell^{1}$ and $\ell^{\infty}$ norms give problems in linear programming. 
The dual (minimum cut) problems will use the dual norms. For $\ell^{2}$ we had the usual BV norm $\iint|\operatorname{grad} u| d x d y$ and the usual measure $|\partial S|=\int d s$ of perimeter. For the $\ell^{1}$ and $\ell^{\infty}$ constraints, the BV norms change and the perimeters reflect those changes (coming from the coarea formula in the new norms):

$$
\begin{aligned}
& |v|_{1} \leq 1 \text { leads to }\|u\|_{\mathrm{BV}}=\iint|\operatorname{grad} u|_{\infty} d x d y \text { and }|\partial S|_{\infty}=\int_{\partial S} \max (|d x|,|d y|) \\
& |v|_{\infty} \leq 1 \text { leads to }\|u\|_{\mathrm{BV}}=\iint|\operatorname{grad} u|_{1} d x d y \text { and }|\partial S|_{1}=\int_{\partial S}|d x|+|d y| .
\end{aligned}
$$

The perimeter of a square changes as the square is rotated, because the norm of $(d x, d y)$ is changing. In each case the dual problem looks for the minimum cut as the solution to a constrained isoperimetric problem:

$$
\begin{aligned}
& \text { Duals of } \\
& \boldsymbol{\ell}^{\mathbf{2}}, \boldsymbol{\ell}^{\mathbf{1}}, \boldsymbol{\ell}^{\infty}
\end{aligned} \underset{S \subset[0,1]^{2}}{\text { Minimize }} \frac{|\partial S|}{|S|} \text { and } \frac{|\partial S|_{\infty}}{|S|} \text { and } \frac{|\partial S|_{1}}{|S|} .
$$

In all cases the optimal $S$ will reach the boundary $\partial \Omega$ of the square. (If $S$ is stretched by a factor $c$, the areas in the denominators of (22) are multiplied by $c^{2}$ and the numerators by $c$.) The symmetry of the problem ensures that the optimal $\partial S$ contains four flat pieces of $\partial \Omega$, centered on the sides of the square (Figure 1). The only parameter in the three optimization problems is the length $L$ of those boundary pieces!

Figure 1a shows the solution for the $\ell^{2}$ problem, where the "unconstrained" parts of the cut $\partial S$ are circular arcs. This follows from the classical isoperimetric problem, and it is easy to show that the arcs must be tangent to the square. The four arcs would fit together in a circle of radius $r$. With $L=1-2 r$, the optimal cut solves the Cheeger problem:

$$
t_{\max }=h(\Omega)=\min \frac{\text { perimeter of } S}{\text { area of } S}=\min \frac{4(1-2 r)+2 \pi r}{1-4 r^{2}+\pi r^{2}} .
$$

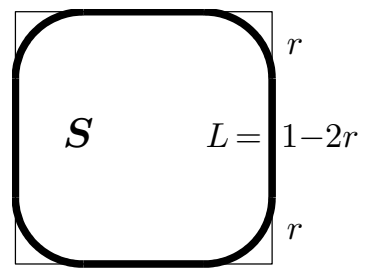

$|\partial S|=4 L+2 \pi r$

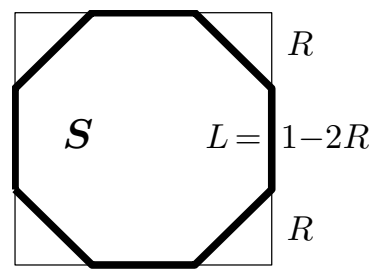

$|\partial S|_{\infty}=4 L+4 R$

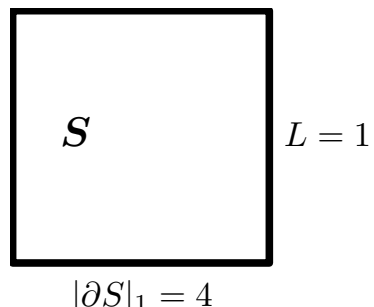

Figure 1: The minimum cuts $\partial S$ for $\ell^{2}, \ell^{1}$, and $\ell^{\infty}$ constraints on $v(x, y)$.

The derivative of that ratio is zero when

$$
\left(1-4 r^{2}+\pi r^{2}\right)(8-2 \pi)=(4-8 r+2 \pi r)(8 r-2 \pi r) .
$$


Cancel $8-2 \pi$ to reach $1-4 r+(4-\pi) r^{2}=0$. Then $r=1 /(2+\sqrt{\pi}) \approx .265$. The Cheeger constant $h(\Omega)$ is the ratio $|\partial S| /|S|=1 / r=2+\sqrt{\pi}$.

A prize of 10,000 yen was offered in [30] for the flow field that achieves $\operatorname{div} v=2+\sqrt{\pi}$ with $|v| \leq 1$. Lippert [20] and Overton [24] have the strongest claim on the prize, by computing a close approximation to $v$. The discrete velocity clearly confirms the cut in Figure 1a as the set where $|v|=1$.

The eigenfunctions of the Laplacian on the unit square are $(\sin \pi x)(\sin \pi y)$ and the lowest eigenvalue is $\lambda_{1}=2 \pi^{2}$. Cheeger's inequality $\lambda_{1} \geq h^{2} / 4$, which other authors have tested earlier, is far from tight:

$$
\text { Unit square } 2 \pi^{2}>(2+\sqrt{\pi})^{2} / 4 \text { or } 19.74>3.56 \text {. }
$$

The second challenge problem has $\left|v_{1}\right|+\left|v_{2}\right| \leq 1$ leading to the measure $|\partial S|_{\infty}$ of the perimeter in the dual. Now the unconstrained isoperimetric problem is solved by a diamond with $\left|n_{1}\right|=\left|n_{2}\right|=1 / \sqrt{2}$ on all edges. The optimal cut $\partial S$ in Figure $1 \mathrm{~b}$ is a union of boundary pieces and diamond edges. The edge length $\sqrt{2} R$ is multiplied by $1 / \sqrt{2}$ from $\left|n_{1}\right|=\left|n_{2}\right|$ to give $|\partial S|_{\infty}=4 L+4 R=4-4 R$. Then the minimum cut has $R=(2-\sqrt{2}) / 2 \approx .3$ :

$$
\min \frac{|\partial S|_{\infty}}{|S|}=\min _{R} \frac{4-4 R}{1-2 R^{2}}=\frac{\sqrt{2}}{\sqrt{2}-1} \approx 3.5
$$

For the flow field $v$ in this $\ell^{1}$ problem, the prize is reduced to 5000 yen. Lippert has reached the computational prize also in $\ell^{1}$. This is linear programming and interior point methods soundly defeated the simplex method.

We cannot afford the prize in the $\ell^{\infty}$ problem, whose solution is simply $v=(2 x-1,2 y-1)$ on the square $0 \leq x, y \leq 1$ with $\operatorname{div} v=4=t_{\max }$.

The minimum cut for the $\ell^{\infty}$ problem is the whole boundary of the square. This coincides with the unconstrained isoperimetric solution when the perimeter is measured by $\int|d x|+|d y|$. The minimizing set $S$ would have horizontal and vertical sides wherever the constraint $S \subset \Omega$ is inactive - and here it is active everywhere on $\partial S=\partial \Omega$. The Cheeger constant in this norm is $h=4 / 1$.

In [32] we prove that the unit ball in the dual norm (rotated by $\pi / 2$ ) is isoperimetrically optimal. Here that ball is a circle or a diamond or a square. This isoperimetrix was discovered by Busemann [5] using the Brunn-Minkowski theorem in convex geometry (the Greeks knew much earlier about the circle).

Our proof finds a simple linear equation for the support function of the optimal convex set $S$.

\section{References}

[1] N. Alon, Eigenvalues and expanders, Combinatorica 6 (1986) 86-96.

[2] B. Appleton and H. Talbot, Globally minimal surfaces by continuous Maximal Flows, IEEE Transactions on Pattern Analysis and Machine Intelligence 28 (2006) 106-118. 
[3] Y. Boykov and V. Kolmogorov, An experimental comparison of mincut/max-flow algorithms for energy minimization in vision, IEEE Trans. Pattern Analysis and Machine Intelligence 26 (2004) 1124-1137.

[4] Y. Boykov, O. Veksler, and R. Zabih, Fast approximate energy minimization via graph cuts, IEEE Transactions on Pattern Analysis and Machine Intelligence 23 (2001) 1222-1239.

[5] H. Busemann, The isoperimetric problem in the Minkowski plane, American J. Math. 69 (1947) 863-871.

[6] J. D. Chavez and L. H. Harper, Duality theorems for a continuous analog of Ford-Fulkerson flows in networks, Adv. in Appl. Math. 14 (1993) 369-388.

[7] J. Cheeger, A lower bound for the smallest eigenvalue of the Laplacian, Problems in Analysis, 1970, 195-199.

[8] F. R. K. Chung, Spectral graph theory, CBMS Regional Conference Series in Mathematics, vol. 92, 1997.

[9] F. R. K. Chung, Laplacians and the Cheeger inequality for directed graphs, Annals of Combinatorics 9 (2005) 1-19.

[10] P. Diaconis and D. W. Stroock, Geometric bounds for eigenvalues of Markov chains, Ann. Appl. Probab. 1 (1991) 36-61.

[11] L. C. Evans, Survey of applications of PDE methods to Monge-Kantorovich mass transfer problems, www . math. berkeley. edu/ evans (earlier version: Current Developments in Mathematics, 1997).

[12] W. Fleming and R. Rishel, An integral formula for total gradient variation, Archiv der Mathematik 11 (1960) 218-222.

[13] L. R. Ford Jr. and D. R. Fulkerson, Flows in Networks, Princeton University Press, 1962.

[14] L. R. Ford Jr. and D. R. Fulkerson, Maximal flow through a network, Canad. J. Math. 8 (1956) 399-404.

[15] W. Gangbo and R. McCann, Optimal maps in Monge's mass transport problem, C.R. Acad. Sci. Paris. Ser. I. Math. 325 (1995) 1653-1658.

[16] D. Grieser, The first eigenvalue of the Laplacian, isoperimetric constants, and the max flow min cut theorem, Archiv der Mathematik, to appear.

[17] T. C. Hu, Integer Programming and Network Flows, Addison-Wesley, 1969.

[18] M. Iri, Theory of flows in continua as approximation to flows in networks, Survey of Mathematical Programming 2 (1979) 263-278.

[19] R. Kohn and R. Temam, Dual spaces of stresses and strains, Appl. Math. and Opt. 10 (1983) 1-35. 
[20] R. Lippert, Discrete approximations to continuum optimal flow problems, Studies in Applied Mathematics, to appear.

[21] J. S. Mitchell, On maximum flows in polyhedral domains, Proc. Fourth Ann. Symp. Computational Geometry, 341-351, 1988.

[22] R. Nozawa, Examples of max-flow and min-cut problems with duality gaps in continuous networks, Mathematical Programming 63 (1994) 213-234.

[23] R. Nozawa, Max-flow min-cut theorem in an anisotropic network, Osaka J. Math. 27 (1990) 805-842.

[24] M. L. Overton, Numerical solution of a model problem from collapse load analysis, Computing Methods in Applied Science and Engineering VI, R. Glowinski and J. L. Lions, eds., Elsevier, 1984.

[25] S. T. Rachev and L. Rüschendorf, Mass Transportation Problems I, II, Springer (1998).

[26] S. Roy and I. J. Cox, A maximum-flow formulation of the n-camera stereo correspondence problem, Proc. Int. Conf. Computer Vision (1988) 492-499.

[27] F. Santosa, An inverse problem in photolithography, in preparation.

[28] R. Sedgewick, Algorithms in C, Addison-Wesley, 2002.

[29] G. Strang, A minimax problem in plasticity theory, in: Nashed, Z. (ed.), Functional Analysis Methods in Numerical Analysis, Lecture Notes in Mathematics 701 (Springer, 1979).

[30] G. Strang, $L^{1}$ and $L^{\infty}$ approximation of vector fields in the plane, Lecture Notes in Num. Appl. Anal. 5 (1982) 273-288.

[31] G. Strang, Maximal flow through a domain, Math. Programming 26 (1983) 123-143.

[32] G. Strang, Maximum area with new measures of perimeter, (2006).

[33] C. Villani, Topics in Optimal Transportation, Graduate Studies in Mathematics 58 (American Mathematical Society, 2003).

This research was supported by the Singapore-MIT Alliance. 